The final version is published in Journal of Surveying Engineering (2020) 146(2):04020008. The material may be downloaded for personal use only. Any other use requires prior permission of the American Society of Civil Engineers. This material may be found at https://ascelibrary.org/doi/full/10.1061/\%28ASCE\%29SU.1943-5428.0000313

\title{
The ADOP and PDOP: Two complementary diagnostics for GNSS positioning
}

\author{
Kan Wang ${ }^{1}$, P.J.G. Teunissen ${ }^{2}$, A. El-Mowafy ${ }^{3}$
}

\section{ABSTRACT}

The Ambiguity Dilution of Precision (ADOP) and the Position Dilution of Precision (PDOP) are two popular scalar-diagnostics used in Global Navigation Satellite System (GNSS) positioning. Where the ADOP is a predictor for carrier-phase ambiguity resolution performance, the PDOP is meant to predict the receiver-satellite geometry's capability for precise positioning. We will show however, although the PDOP works well for code-based positioning, that one has to exercise great care in using the PDOP for real-time kinematic (RTK) positioning. We show that the ADOP and PDOP have distinct behaviors, an important consequence of which is that one can have time periods with small PDOPs, and thus seemingly good geometry for precise positioning, but at the same time large ADOPs, thus showing that successful ambiguity resolution and therefore precise positioning will not be possible. Also the reverse situation may occur, i.e. having large PDOPs with small ADOPs. In such a situation, the large PDOPs should not automatically lead to the conclusion of poor position performance, since the large gain that ambiguity resolution brings will often still make precise positioning possible. We will analyse and explain this complementary behavior of the PDOP and ADOP, and demonstrate this both analytically and empirically. For this analysis we use real Global Positioning System (GPS) single- and multi-frequency signals and GPS/Quasi-Zenith Satellite System (QZSS), GPS/Navigation with Indian Constellation (NAVIC) L5 signals of two baselines located in Perth, Australia.

Keywords: Position Dilution of Precision (PDOP), Ambiguity Dilution of Precision (ADOP), Ambiguity Success-Rate (ASR), Integer Ambiguity Resolution (IAR), Real-Time Kinematic (RTK), Instantaneous Positioning.

\section{INTRODUCTION}

The Ambiguity Dilution of Precision (ADOP) and the Position Dilution of Precision (PDOP) are two popular scalar-diagnostics used in Global Navigation Satellite System (GNSS) positioning (Langley 1999, Gleason and Gebre-Egziabher 2009, Liu et al. 2017). The PDOP is an easy-to-compute diagnostic to predict the impact of the receiver-satellite geometry on the precision of positioning. Although originally first used for Global Posi-tioning System (GPS) single-point positioning (Bogen 1974, Spilker 1996), the PDOP has found its usage in a broad range of GNSS positioning applications (Betz 2016, Teunissen and Montenbruck 2017). The ADOP is an easy-to-compute diagnostic to predict the success

\footnotetext{
${ }^{1}$ Research Associate, School of Earth and Planetary Sciences, Curtin University, GPO Box U1987, Perth, WA 6845, Australia (corresponding author). E-mail: kan.wang@curtin.edu.au

${ }^{2}$ Professor, School of Earth and Planetary Sciences, Curtin University, GPO Box U1987, Perth, WA 6845, Australia; Department of Geoscience and Remote Sensing, Delft University of Technology, 2628 CN Delft, The Netherlands. E-mail: P.Teunissen@curtin.edu.au

${ }^{3}$ Associate Professor, School of Earth and Planetary Sciences, Curtin University, GPO Box U1987, Perth, WA 6845, Australia. E-mail: A.El-Mowafy@curtin.edu.au
} 
of carrier-phase integer ambiguity resolution. Although originally introduced for GPS ambiguity resolution (Teunissen 1997), the ADOP has found its usage in various contemporary GNSS precise positioning applications (Geng and Bock 2013, Odolinski et al. 2015, Li et al. 2016, Liu et al. 2019).

In this contribution we will study the PDOP and ADOP in their mutual relation, analytically as well as empirically, and show that their distinct characteristics imply that one has to exercise care in using the PDOP as a sole-means predictor for enabled precise positioning. We show that a small PDOP may not necessarily be good for real-time kinematic (RTK) positioning, while a large PDOP may not be necessarily bad for such positioning. We also demonstrate this with real data. By highlighting the complementary characteristics of the PDOP and ADOP, we show that they should be used in tandem in order to have a realistic predictability of precise positioning. As nowadays many commercial relative positioning software reports PDOP as an indicator for the positioning precision, but rarely reports ADOP to help users when planning and executing their GNSS work, this paper attempts to analytically and empirically distinguish the natures of the PDOP and ADOP, and show the importance of reporting ADOP in addition. As PDOP, we believe ADOP should also play a predictive role for planning the GNSS work in the actual world.

This contribution is organized as follows. In section 2 we first present our model formulation which forms the basis of our analyses. Then in section 3 we describe our measurement set-up and present our signal analysis. The data used comprises real GPS singleand multi-frequency signals, as well as GPS/Quasi-Zenith Satellite System (QZSS) and GPS/Navigation with Indian Constellation (NAVIC) L5 signals of two baselines located in Perth, Australia. In section 4 we discuss the PDOP, analyse its characteristics and in particular show that it can be used to obtain a very good approximation to the average RTK ambiguity-resolved baseline standard deviation. We use real data to validate this by showing the good match between formal and empirically determined standard deviations. Note that here 'formal' means the computation based on geometry and model, but not based on the real data. In section 5 we discuss the ADOP, analyse its characteristics and in particular explain why it provides a good approximation to the probability of correct integer ambiguity estimation, i.e., ambiguity success-rate. We then demonstrate in this section that the ADOP and PDOP have very distinct characteristics. We explain this difference and show that as a consequence one should take great care in using the PDOP as sole-means predictor for precise positioning capability. In section 6 we then substantiate the formal findings of the previous section with a real-data-based empirical evaluation, thereby showing the complementary benefit of PDOP and ADOP for precise positioning. This contribution is then finalized with our conclusions in section 7 .

\section{MODEL FORMULATION}

The multi-frequency GNSS, linearized short-baseline double-differenced (DD) single-epoch observed-minus-computed (O-C) code $(\boldsymbol{p})$ and phase $(\boldsymbol{\phi})$ observation equations can be formulated as:

$$
\mathrm{E}\left[\begin{array}{l}
\boldsymbol{p} \\
\boldsymbol{\phi}
\end{array}\right]=\left[\begin{array}{ll}
\boldsymbol{D}_{m}^{\mathrm{T}} \boldsymbol{A} & \mathbf{0} \\
\boldsymbol{D}_{m}^{\mathrm{T}} \boldsymbol{A} & \boldsymbol{\Lambda}
\end{array}\right]\left[\begin{array}{l}
\boldsymbol{b} \\
\boldsymbol{a}
\end{array}\right]
$$

where $\boldsymbol{b}$ denotes the baseline increment vector in units of distance and $\boldsymbol{a}$ the DD ambiguity 
vector in cycles. E[·] is the expectation operator. The terms $m, \boldsymbol{D}_{m}^{\mathrm{T}}, \boldsymbol{\Lambda}$ and $\boldsymbol{A}$ are given as

$$
\begin{aligned}
m & =\sum_{j=1}^{f} \mu_{j} \\
\boldsymbol{D}_{m}^{\mathrm{T}} & =\operatorname{blkdiag}\left(\boldsymbol{D}_{\mu_{1}}^{\mathrm{T}}, \cdots, \boldsymbol{D}_{\mu_{f}}^{\mathrm{T}}\right) \\
\boldsymbol{\Lambda} & =\operatorname{blkdiag}\left(\lambda_{1} \boldsymbol{I}_{\mu_{1}-1}, \cdots, \lambda_{f} \boldsymbol{I}_{\mu_{f}-1}\right) \\
\boldsymbol{A} & =\left[\boldsymbol{v}^{1}, \cdots, \boldsymbol{v}^{m}\right]^{\mathrm{T}}
\end{aligned}
$$

for which $f$ denotes the number of frequencies, and $\mu_{j}$ denotes the number of satellites above the elevation mask transmitting signals on frequency $j$. Note that $m$ here denotes the sum of $\mu_{j}$ over all frequencies, i.e., the total number of phase or code observations, but not the number of satellites. The same satellite transmitting signals on different frequencies would thus have different indices in $\boldsymbol{v}^{s}(s=1, \cdots, m)$, which is the satellite-to-receiver unit vector for the corresponding observation. $\boldsymbol{D}_{\mu_{j}}^{\mathrm{T}}$ is the differencing operator with $\boldsymbol{D}_{\mu_{j}}^{\mathrm{T}}=$ $\left[-\boldsymbol{e}_{\mu_{j}-1}, \boldsymbol{I}_{\mu_{j}-1}\right]$, where $\boldsymbol{e}_{\mu_{j}-1}$ and $\boldsymbol{I}_{\mu_{j}-1}$ are the vector of ones with the size $\mu_{j}-1$ and the identity matrix with the size $\left(\mu_{j}-1\right) \times\left(\mu_{j}-1\right)$, respectively. The wavelength on frequency $j$ is denoted by $\lambda_{j}$, and blkdiag(.) forms a block diagonal matrix using the matrices contained in $(\cdot)$. Here, on the same frequency, multi-GNSS satellites could be included in the observation model. In this study, we assume the differential inter-system biases (ISBs) to be absent, since the stations forming the baselines in our study used the same type of receiver and antenna (Odijk et al. 2017). We therefore select for each frequency and each epoch, one reference satellite for all satellites of all systems. The Multi-GNSS Experiment (MGEX) combined broadcast ephemeris (Montenbruck et al. 2014, Montenbruck et al. 2017, MGEX 2018) was used to compute satellite orbits.

Based on Eq. 1, the single-epoch variance-covariance matrix of $\boldsymbol{p}$ and $\boldsymbol{\phi}$ can be formulated as

$$
\mathrm{D}\left[\begin{array}{l}
\boldsymbol{p} \\
\boldsymbol{\phi}
\end{array}\right]=\left[\begin{array}{cc}
2 \boldsymbol{D}_{m}^{\mathrm{T}} \boldsymbol{Q}_{\boldsymbol{p} \boldsymbol{p}} \boldsymbol{D}_{m} & \mathbf{0} \\
\mathbf{0} & 2 \boldsymbol{D}_{m}^{\mathrm{T}} \boldsymbol{Q}_{\boldsymbol{\phi} \phi} \boldsymbol{D}_{m}
\end{array}\right]
$$

with

$$
\begin{aligned}
& \boldsymbol{Q}_{\boldsymbol{p} \boldsymbol{p}}=\operatorname{diag}\left(\sigma_{p_{j k(1)}^{2}}^{2}, \cdots, \sigma_{p_{j k(m)}}^{2}\right) \boldsymbol{W}^{-1} \\
& \boldsymbol{Q}_{\boldsymbol{\phi} \phi}=\operatorname{diag}\left(\sigma_{\phi_{j k(1)}}^{2}, \cdots, \sigma_{\phi_{j k(m)}}^{2}\right) \boldsymbol{W}^{-1}
\end{aligned}
$$

where $\operatorname{diag}(\cdot)$ forms the diagonal matrix with the diagonal elements contained in $(\cdot)$, and $\mathrm{D}(\cdot)$ is the dispersion operator. The terms $\sigma_{p_{j k(s)}}$ and $\sigma_{\phi_{j k(s)}}$ are zenith-referenced code and phase signal standard deviations of frequency $j$ and system $k$ assigned for the $s$-th signal, respectively. The elevation-dependent weight matrix $\boldsymbol{W}$ is given as (Euler and Goad 1991)

$$
\boldsymbol{W}=\operatorname{diag}\left(w^{1}, \cdots, w^{m}\right)
$$

with

$$
w^{s}=\left(1+10 \times \exp \left(-\frac{e^{s}}{10^{\circ}}\right)\right)^{-2}
$$

where $e^{s}$ is the elevation angle from the receiver to satellite in degrees, and $\exp (\cdot)$ is the natural exponential function. The elevation cut-off angle is set to 10 degrees. Note that 
for the short RTK baselines considered here, the elevation angles from both receivers to the same satellite are assumed to be the same.

As mentioned above, in this contribution, our analysis will be based on both a singleGNSS multi-frequency scenario as well as a multi-GNSS single-frequency scenario. We use an epoch-by-epoch processing whereby the ambiguity parameters are estimated independently for each epoch and resolved with the least-squares ambiguity decorrelation adjustment (LAMBDA) method (Teunissen 1995) using the integer bootstrapping (IB) estimator. In the next section, the measurement set up is introduced together with a signal analysis for the different GNSSs and their different frequencies.

\section{MEASUREMENT SET UP AND SIGNAL ANALYSIS}

In this study, two baselines located at Curtin University, Perth, Australia, were used for the data analysis. The first baseline CUAA-CUBB has a length of about $9 \mathrm{~m}$ and the second baseline CUCC-SPA7 has a length of about $352 \mathrm{~m}$. All four stations use the same type of receiver, i.e., JAVAD TRE_G3TH DELTA (Javad, San Jose, CA, USA) and the same type of antenna, i.e., TRM59800.00 SCIS (Trimble, Sunnyvale, CA, USA). The data sampling rate is $1 \mathrm{~Hz}$.

As mentioned before, in this study, the GPS single- (L1), dual- (L1, L2) and triplefrequency (L1, L2, L5) scenarios and the L5 multi-GNSS scenarios were used for the data analysis. The latter case includes the L5 GPS/QZSS and the L5 GPS/NAVIC scenarios. Figure 1 shows the skyplots based on the satellite geometry for station CUAA with different scenarios on Day of Year (DOY) 328 and 343, 2018. In Figure 1a, 12 GPS IIF satellites marked with dashed lines were transmitting L1, L2 and L5 signals, while 18 other GPS satellites from blocks IIR and IIR-M were transmitting signals on L1 and L2. Note that the only operational GPS IIA satellite G18 was not contained in the combined MGEX broadcast ephemeris on DOY 328, 2018. Although not plotted in Figure 1a nor used in this study, it is remarked that the first GPS Block IIIA satellite was launched on December 23, 2018. From Figure $1 \mathrm{~b}$ it can be observed that 4 QZSS satellites and 6 NAVIC satellites transmitting L5 signals were also visible from CUAA. Among the four QZSS satellites, J07 is a geostationary (GEO) satellite, while the other three QZSS satellites are in quasi-zenith orbits (QZOs). Among the 6 visible NAVIC satellites, I03 and I07 are GEO satellites, while I02, I04, I05 and the newly launched I09 in April 2018 (ISRO 2018) are in inclined geosynchronous orbits (IGSOs).

In order to minimize the effect of multipath on our analyses, the processing was performed after multipath-mitigation. Accordingly, as the repeat cycles of the GPS, NAVIC and QZSS satellites are around 1 sidereal day, the multipath-mitigated DD code $(\boldsymbol{d} \boldsymbol{p})$ and phase $(\boldsymbol{d} \boldsymbol{\phi})$ residuals were computed for each baseline at epoch $t_{i}$ by forming day-to-day differences:

$$
\left[\begin{array}{l}
\boldsymbol{d} \boldsymbol{p}\left(t_{i}\right) \\
\boldsymbol{d} \boldsymbol{\phi}\left(t_{i}\right)
\end{array}\right]=\left[\begin{array}{l}
\boldsymbol{p}\left(t_{i}\right) \\
\boldsymbol{\phi}\left(t_{i}\right)
\end{array}\right]-\left[\begin{array}{l}
\boldsymbol{p}\left(t_{i}+T\right) \\
\boldsymbol{\phi}\left(t_{i}+T\right)
\end{array}\right]-\left[\begin{array}{ll}
\boldsymbol{I}_{m-f} & \mathbf{0} \\
\boldsymbol{I}_{m-f} & \boldsymbol{\Lambda}
\end{array}\right]\left[\begin{array}{l}
\boldsymbol{\rho}\left(t_{i}\right)-\boldsymbol{\rho}\left(t_{i}+T\right) \\
\check{\boldsymbol{a}}\left(t_{i}\right)-\check{\boldsymbol{a}}\left(t_{i}+T\right)
\end{array}\right]
$$

where $T$ denotes the repeat cycle of the satellites, i.e., $23 \mathrm{~h} 56 \mathrm{~min}$ (which is of sufficient accuracy for this application), and $\boldsymbol{\rho}$ denotes the DD geometry computed based on the satellite orbits and ground truth of both receivers. The fixed day-to-day DD ambiguities

$\check{\boldsymbol{a}}\left(t_{i}\right)-\check{\boldsymbol{a}}\left(t_{i}+T\right)$ were computed based on the strong baseline-known model, in which the receiver coordinates are fixed to known values (Zaminpardaz et al. 2018, Wang et al. 2019). 
With the DD phase and code residuals, the zenith-referenced standard deviations were computed for each baseline and signal type with the least-squares variance component estimation (LS-VCE) procedure (Amiri-Simkooei et al. 2009). The computed code and phase signal standard deviations along the zenith direction, denoted as $\sigma_{p}$ and $\sigma_{\phi}$, respectively, are given in Table 1 and used in the processing. The data on DOY 325/326 and on DOY 328/329 were used for the calculation and multipath mitigation of GPS signals for baselines CUAA-CUBB and CUCC-SPA7, respectively. Additionally, the data on DOY 345/346 and 335/336, 2018 were used for analysis of the QZSS signals for baselines CUAA-CUBB and CUCC-SPA7, respectively, and the observations from the former two days were used for analysis of the NAVIC signals of both baselines. Note that the factor of $\sqrt{2}$ when forming the day-to-day differences was included in the standard deviations that are given in Table 1.

\section{THE POSITION DILUTION OF PRECISION: PDOP}

The PDOP is a popular scalar diagnostic to infer the impact of the receiver-satellite geometry on the precision of GNSS positioning (Bogen 1974, Langley 1999). In the context of GNSS it was first used for GPS code-based single-point positioning (SPP), whereby the PDOP values could give a general overview of the daily positioning precision and its changes with time (Spilker 1996). Also other types of DOP-values exist in addition to the PDOP, like e.g. GDOP (geometric), HDOP (horizontal), or VDOP (vertical). For SPP, with $\left[\boldsymbol{A}, \boldsymbol{e}_{m}\right]$ being its design matrix, all of these DOP values are based on different subsets of the diagonal entries of the matrix, see Section 1.2.4 in (Teunissen and Montenbruck 2017)

$$
\left(\left[\boldsymbol{A}, \boldsymbol{e}_{\boldsymbol{m}}\right]^{\mathrm{T}} \boldsymbol{W}\left[\boldsymbol{A}, \boldsymbol{e}_{\boldsymbol{m}}\right]\right)^{-1}=\left[\begin{array}{cccc}
q_{n n} & q_{n e} & q_{n h} & q_{n t} \\
q_{e n} & q_{e e} & q_{e h} & q_{e t} \\
q_{h n} & q_{h e} & q_{h h} & q_{h t} \\
q_{t n} & q_{t e} & q_{t h} & q_{t t}
\end{array}\right]
$$

With the first three diagonal entries of this matrix corresponding to north $(n)$, east $(e)$ and height $(h)$, the PDOP is defined as (Zaminpardaz et al. 2018)

$$
\begin{aligned}
P D O P & =\sqrt{q_{n n}+q_{e e}+q_{h h}} \\
& =\sqrt{\operatorname{tr}\left(\left(\boldsymbol{A}^{\mathrm{T}} \boldsymbol{P}_{\boldsymbol{D}_{\boldsymbol{m}}} \boldsymbol{W} \boldsymbol{A}\right)^{-1}\right)}
\end{aligned}
$$

with projector $\boldsymbol{P}_{\boldsymbol{D}_{m}}=\boldsymbol{D}_{m}\left(\boldsymbol{D}_{m}^{\mathrm{T}} \boldsymbol{W}^{-1} \boldsymbol{D}_{m}\right)^{-1} \boldsymbol{D}_{m}^{\mathrm{T}} \boldsymbol{W}^{-1}$ and where $\operatorname{tr}(\cdot)$ represents the trace of the matrix contained in $(\cdot)$. Note that the second expression in (13) follows from having the normal matrix $\left[\boldsymbol{A}, \boldsymbol{e}_{m}\right]^{\mathrm{T}} \boldsymbol{W}\left[\boldsymbol{A}, \boldsymbol{e}_{m}\right]$ reduced for the column of the receiver clock error. Also note that $\boldsymbol{D}_{m}^{\mathrm{T}}$ and $\boldsymbol{W}$ refer to the differencing operator (Eq. 3) and the weight matrix (Eq. 9) in single-frequency scenario.

In addition to SPP, the PDOP represents also in the context of RTK a good scalarindicator for the impact of the receiver-satellite geometry on positioning. Assuming the ambiguities are known, it follows from solving (1) in a least-squares sense that the variancecovariance matrix of the ambiguity-fixed baseline is given as

$$
\begin{aligned}
\boldsymbol{Q}_{\check{\boldsymbol{b}} \check{\boldsymbol{b}}} & =2\left(\boldsymbol{A}^{\mathrm{T}}\left[\boldsymbol{R}_{\boldsymbol{D}_{m}} \boldsymbol{Q}_{\boldsymbol{p} \boldsymbol{p}}^{-1}+\boldsymbol{S}_{\boldsymbol{D}_{m}} \boldsymbol{Q}_{\boldsymbol{\phi} \boldsymbol{\phi}}^{-1}\right] \boldsymbol{A}\right)^{-1} \\
& \approx 2 \sigma_{\bar{\phi}}^{2}\left(\boldsymbol{A}^{\mathrm{T}} \boldsymbol{P}_{\boldsymbol{D}_{m}} \boldsymbol{W} \boldsymbol{A}\right)^{-1}
\end{aligned}
$$


with projectors $\boldsymbol{R}_{\boldsymbol{D}_{m}}=\boldsymbol{D}_{m}\left(\boldsymbol{D}_{m}^{\mathrm{T}} \boldsymbol{Q}_{\boldsymbol{p} \boldsymbol{p}} \boldsymbol{D}_{m}\right)^{-1} \boldsymbol{D}_{m}^{\mathrm{T}} \boldsymbol{Q}_{\boldsymbol{p} \boldsymbol{p}}$ and $\boldsymbol{S}_{\boldsymbol{D}_{m}}=\boldsymbol{D}_{m}\left(\boldsymbol{D}_{m}^{\mathrm{T}} \boldsymbol{Q}_{\boldsymbol{\phi} \boldsymbol{\phi}} \boldsymbol{D}_{m}\right)^{-1} \boldsymbol{D}_{m}^{\mathrm{T}} \boldsymbol{Q}_{\boldsymbol{\phi} \boldsymbol{\phi}}$, and whereby the approximation in Eq. 14 follows from using $\boldsymbol{Q}_{\boldsymbol{p} \boldsymbol{p}}^{-1}<<\boldsymbol{Q}_{\phi \boldsymbol{\phi}}^{-1}$ (see Table 1) and $\sigma_{\bar{\phi}}=\sqrt{\sum_{s=1}^{m} \sigma_{\phi_{j k(s)}}^{2} / m}$. If we now define the average ambiguity-fixed baseline standard deviation as $\sigma_{\breve{b}}=\sqrt{\left(\sigma_{\check{N}}^{2}+\sigma_{\breve{E}}^{2}+\sigma_{\breve{H}}^{2}\right) / 3}$, in the single-frequency case, it follows from combining (13) with (14) that

$$
\sigma_{\breve{b}} \approx P D O P \cdot \sigma_{\bar{\phi}} \cdot \sqrt{\frac{2}{3}}
$$

For multi-frequency scenario, the right side of Eq. 15 is to be divided by, e.g., $\sqrt{f}$ in case that the same satellites are tracked on each frequency.

Figure 2 shows, for the baseline CUCC-SPA7 on DOY 343, 2018, a representative example of how well this approximation works for the L5 multi-GNSS case. With $\sigma_{\bar{\phi}}$ around $1-2 \mathrm{~mm}$ (Table 1), PDOPs below 12 or 6 would approximately correspond to an average formal baseline precision below 2 or $1 \mathrm{~cm}$.

As (15) is concerned with the model-based formal precision, we now verify how well the formal precision matches the data-based empirically determined precision. In Table 2, the time-averaged formal standard deviations of the ambiguity-fixed baseline are shown together with their empirically determined counterparts, i.e., the empirical standard deviations of the ambiguity-fixed baseline errors in north, east and height directions. The data on DOY 328/329 and DOY 325/326, 2018 were used for processing and multipath mitigation in GPS single- and multi-frequency scenarios for baselines CUAA-CUBB and CUCC-SPA7, respectively. The data on DOY 343/344, 2018 were used for the L5 multi-GNSS scenarios for both baselines. The good correspondence between the formal and empirical standard deviations indicates the good correspondence between our model and the data.

\section{THE AMBIGUITY DILUTION OF PRECISION: ADOP}

In single-epoch RTK processing, successfully resolving the integer ambiguities is crucial for improving the baseline precision to the mm-cm level. While PDOP reflects the influence of the receiver-satellite geometry on the ambiguity-fixed baseline precision, it is essential to first be able to assess when, where and under which processing scenarios the ambiguities can be successfully resolved. The ADOP is a popular scalar-diagnostic that predicts such capability of the employed measurement scenario. The ADOP, introduced by Teunissen (1997), is an easy-to-compute scalar diagnostic that measures the intrinsic model strength for successful ambiguity resolution. It is defined as

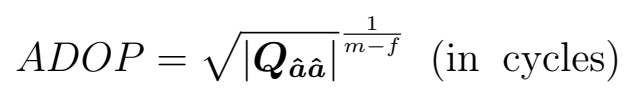

with $\boldsymbol{Q}_{\hat{\boldsymbol{a}} \hat{\boldsymbol{a}}}$ the variance-covariance matrix of the least-squares estimated ambiguities and $|\cdot|$ denoting the matrix determinant. Different from the PDOP, the determinant but not the trace is used to compute the ADOP. As discussed in Teunissen (1997), this is on the one hand because of the lack of Z-invariance when using the trace of $\boldsymbol{Q}_{\hat{a} \hat{a}}$, and on the other hand because the high correlation among the ambiguities cannot be taken into consideration when using the trace, which is important for the ambiguity resolution. As $\boldsymbol{Q}_{\hat{\boldsymbol{a}} \hat{\boldsymbol{a}}}$ is a square matrix with the size $(m-f) \times(m-f)$, raising the inverse power of $m-f$ to $\sqrt{\left|\boldsymbol{Q}_{\hat{\boldsymbol{a}} \hat{\boldsymbol{a}}}\right|}$ gives the ADOP in units of cycles. 
The ADOP has several important properties. First, it is invariant against the choice of ambiguity parametrization. Since all admissible ambiguity transformations can be shown to have a determinant of one, the ADOP does not change when one changes the definition of the ambiguities. It is thus an intrinsic measure independent of the arbitrary choice of ambiguity parametrization. Second, the ADOP is also a measure of the probability mass of the ambiguity confidence ellipsoid and corresponding ambiguity search space (Teunissen et al. 1996). And third, the ADOP equals the geometric mean of the standard deviations of the ambiguities, in the case when the ambiguities are completely decorrelated. Since the LAMBDA method (Teunissen 1995) produces ambiguities that are largely decorrelated, the ADOP approximates the average precision of the transformed ambiguities. It therefore also provides for a good approximation to the integer least-squares (ILS) probability of correct integer estimation $P\left(\check{\boldsymbol{a}}_{\mathbf{I L S}}=\boldsymbol{a}\right)$, i.e., the ILS ambiguity success rate (Odijk and Teunissen 2008):

$$
P\left(\check{\boldsymbol{a}}_{\mathrm{ILS}}=\boldsymbol{a}\right) \approx\left[2 \Phi\left(\frac{1}{2 A D O P}\right)-1\right]^{m-f}
$$

in which $\check{\boldsymbol{a}}_{\text {ILS }}$ is the ILS ambiguity estimator of $\boldsymbol{a}$ and where $\Phi(\cdot)$ is the standard normal cumulative distribution function. From this approximation it follows that one can use $A D O P=0.12$ cycles as a rule of thumb for an ambiguity success rate (ASR) of $99.9 \%$ (Odijk and Teunissen 2008).

We will now show that the ADOPs can have very distinct time behaviors when compared to those of PDOP. Figure 3 shows the PDOP and ADOP patterns in the GPS L1 case. The data of the baseline CUAA-CUBB on DOY 328 were used for the plots, and the ADOP values below 0.12 cycles are marked with circles. From Figure 3 it can be observed that between about $76400 \mathrm{~s}$ and $81800 \mathrm{~s}$, the ADOP jumps to high values, while the PDOPs remain relatively low, i.e., below 4. Thus here the PDOPs describes a receiver-satellite geometry which is good for precise positioning, but the ADOPs make clear that such a case is not achievable due to the poor ambiguity resolution capability over these time-periods.

More distinct PDOP and ADOP patterns can be observed in Figure 4. GPS/QZSS and GPS/NAVIC combined L5 signals from baseline CUCC-SPA7 on DOY 343, 2018 were used for the plots. The ADOP values below 0.12 cycles are marked with dotted lines. From Figure $4 \mathrm{a}$ it can be observed that time periods with large ADOPs at around $4700 \mathrm{~s}$, could have comparably small PDOPs below 5. The sharply decreasing PDOPs from about $78000 \mathrm{~s}$ to $81000 \mathrm{~s}$ correspond to rising ADOPs during this time period. In Figure 4b, it can be seen that PDOPs rose sharply from about $20200 \mathrm{~s}$ to $22800 \mathrm{~s}$ and drop from then to about $26200 \mathrm{~s}$ with PDOP varying from about 4.1 to 10.7. During this time period, ADOP decreases from about 0.17 cycles to below 0.12 cycles. Thus here we have relatively large PDOPs, while the ADOPs show that ambiguity resolution is possible.

To understand the above shown behavior of the ADOPs, we will now have a closer look at the constituents of the ADOP. From the determinant factorization rule (Teunissen et al. 2006), also formulated in Odijk and Teunissen (2008), it follows that $\left|\boldsymbol{Q}_{\breve{b} \breve{b}}\right|\left|\boldsymbol{Q}_{\hat{a} \hat{a} \boldsymbol{a}}\right|=$ $\left|\boldsymbol{Q}_{\hat{a} \hat{a} \mid \boldsymbol{b}}\right|\left|\boldsymbol{Q}_{\hat{b} \hat{b}}\right|$, where $\boldsymbol{Q}_{\hat{a} \hat{a} \mid \boldsymbol{b}}$ represents the variance-covariance matrix of the ambiguities when the baseline is fixed. Since $\boldsymbol{Q}_{\hat{a} \hat{a} \mid \boldsymbol{b}}=2 \boldsymbol{\Lambda}^{-1}\left(\boldsymbol{D}_{m}^{\mathrm{T}} \boldsymbol{Q}_{\phi \phi} \boldsymbol{D}_{m}\right) \boldsymbol{\Lambda}^{-1}$ (see Eqs. 1 and 6), it follows with $\bar{\lambda}=|\boldsymbol{\Lambda}|^{\frac{1}{m-f}}=\left(\prod_{i=1}^{f} \lambda_{i}^{\mu_{i}-1}\right)^{\frac{1}{m-f}}$ being the geometric average of the wavelengths and $\bar{q}_{\phi}=\sqrt{\left|\left(\boldsymbol{D}_{m}^{\mathrm{T}} \boldsymbol{Q}_{\phi \phi} \boldsymbol{D}_{m}\right)\right|^{\frac{1}{m-f}}}$ the geometric average of the DD phase precision, that the ADOP 
can be expressed as

$$
A D O P=\sqrt{2} \frac{\bar{q}_{\phi}}{\bar{\lambda}}\left(\frac{\sqrt{\left|\boldsymbol{Q}_{\hat{b} \hat{b}}\right|}}{\sqrt{\left|\boldsymbol{Q}_{\bar{b} \tilde{b}}\right|}}\right)^{\frac{1}{m-f}}
$$

This shows that the ADOP is a measure of the change in baseline precision before and after ambiguity-fixing and thus not, as with the PDOP, a measure of the geometric impact on the baseline precision itself. This explains the distinct time-behaviors of the ADOPs and PDOPs. An important consequence of this difference is that one can have time periods with small PDOPs, and thus seemingly good geometry for precise positioning, but at the same time large ADOPs, thus showing that successful ambiguity resolution and therefore precise positioning will not be possible. This shows that without the ADOP, one cannot rely on the PDOP for predicting precise positioning capabilities, in single-epoch RTK for instance. This also holds true for the reverse situation, when one has time periods with small ADOPs but large PDOPs. It is common practice to discard positioning when the PDOPs are larger than 10, however when small ADOPs are present, successful ambiguity resolution could still give in such situations good enough positioning results. A PDOP of 20, for instance, may still produce an average baseline precision of around $3 \mathrm{~cm}$. The important conclusion is therefore that for the predictability of precise positioning in single-epoch RTK processing, the evaluation of the ADOPs should take priority over the PDOP evaluation.

\section{AN EMPIRICAL EVALUATION}

In this section we will make an empirical evaluation, thereby substantiating our ADOPPDOP findings of the previous sections. First we will compare the empirical success-rates with their formal counterparts. As we performed ambiguity resolution with integer bootstrapping (IB), the formal ambiguity success-rate (ASR), denoted as $P_{I B}$, is given by (Teunissen 1998):

$$
P_{I B}=\prod_{i=1}^{m-f}\left(2 \Phi\left(\frac{1}{2 \sigma_{\hat{z}_{i \mid I}}}\right)-1\right)
$$

in which $\sigma_{\hat{z}_{i \mid I}}$ is the $i$-th conditional standard deviation of the ambiguities after decorrelation with $I=1, \cdots, i-1$. The correspondence between the time-averaged formal-ASR (based on (19)) and the empirical-ASR is shown in Table 3 for both baselines used in our tests. The empirical-ASR is hereby obtained as $P_{E}=\frac{N_{C}}{N}$ where $N_{C}$ and $N$ denote the number of time epochs with correctly fixed ambiguities and the total number of epochs, respectively. The decision whether or not the single-epoch resolved ambiguities were correct or not was made by comparing them to reference ambiguities which were obtained using the baseline-known model (Zaminpardaz et al. 2018, Wang et al. 2019). As Table 3 shows, there is a very good agreement between the formal and empirical results.

We now show our empirical coordinate time-series (float and ambiguity-fixed) and their relation to the ADOPs and PDOPs. Figure 5 shows the north, east and height errors for baseline CUCC-SPA7 on DOY 343, 2018 using GPS/NAVIC L5 observations. The processing was performed based on the observation model described in Section 2. The data on DOY 344, 2018 were used for multipath mitigation by forming day-to-day differences of the DD observations, and with the known DD receiver-satellite distance on the DOY 344, 2018 removed. The dots (the first item in the legend), solid lines (the third item in the legend) and 
$\mathrm{x}$-marks (the fourth item in the legend) illustrate the ambiguity-float, ambiguity-correctlyfixed and ambiguity-wrongly-fixed solutions with the values given at the left side of the $\mathrm{y}$-axis. The dotted lines (the second item in the legend) indicate the $95 \%$ formal confidence intervals. The pentagrams (the fifth item in the legend) represent the ADOP values on the same day with their values given at the right side of the y-axis. The dashed lines (the last item in the legend) mark an ADOP of 0.12 cycles. From Figure 5 it can be observed that the ambiguity-float baseline errors have a range up to a few metres with standard deviations at a few decimetres to around $1 \mathrm{~m}$. The average formal and empirical standard deviations correspond well with each other. Also we see the predictability of the ADOP clearly at work. Wrongly fixed ambiguities predominantly happen in the time-intervals for which ADOP $>0.12$ cycles. And this is not only seen in Figure 5, but also in Figure 6, which is based on using GPS L1 signals of baseline CUAA-CUBB on DOY 328, 2018.

We can also demonstrate that the time periods with frequent wrongly fixed ambiguities (see the x-marks in Figure 5) do not necessarily have larger PDOPs. The time period having the most x-marks in Figure 5, i.e., before about $1500 \mathrm{~s}$, from about $17700 \mathrm{~s}$ to $20200 \mathrm{~s}$, and from about $78000 \mathrm{~s}$ to $81000 \mathrm{~s}$, do not necessarily have the largest PDOP over the day (Figure 4b). The highest PDOPs appear between about $22300 \mathrm{~s}$ and $23200 \mathrm{~s}$, which also corresponds to the large ambiguity-fixed height errors. The ADOP during this time period is only slightly above 0.12 cycles with the ambiguities mostly correctly fixed.

Figure 7 shows the ambiguity-correctly-fixed solutions (dots, the first item in the legend) with the corresponding 95\% formal confidence intervals bounded with dotted lines. The PDOPs are shown as pentagrams (the third item in the legend) with values given at the right side of the y-axis. Again note that the time-period having the peak with the large PDOP still has good capabilities for precise positioning within a few centimetre accuracy. Similarly, we see in Figure 8 the ambiguity-correctly-fixed solutions for baseline CUAACUBB using GPS L1 signals with the PDOP plotted at the right side of the axis. As observed from Figure 8, the PDOPs in the GPS L1-only case are mostly between 3 and 4 for the tested baseline and do not vary much during the entire day. And again, during the time period with large ADOPs between about $76400 \mathrm{~s}$ and $81800 \mathrm{~s}$ (see Figure 6), PDOPs are not observed to be especially large in Figure 8.

\section{CONCLUSIONS}

In this contribution we studied the PDOP and ADOP in their mutual relation. We showed, both formally and empirically, that they have distinct characteristics and that a good understanding of this difference is important for their proper usage in the context of precise positioning. Their distinct time-behaviors were explained by showing that the ADOP is a measure of the change in baseline precision before and after ambiguity-fixing, whereas the PDOP is a measure of the geometric impact on the baseline precision itself.

An important consequence of this difference is that one can have time periods with small PDOPs, and thus seemingly good geometry for precise positioning, but at the same time large ADOPs, thus showing that successful ambiguity resolution and therefore precise positioning will not be possible. Similarly, we showed that a large PDOP may not necessarily be bad for such positioning if the ADOP is small. The conclusion reads therefore that for single-epoch RTK positioning, without the ADOP, one cannot rely on the PDOP for predicting precise positioning capabilities. As they are complementary, the ADOP and PDOP have to be used 
in tandem in order to have a realistic predictability of precise positioning.

\section{ACKNOWLEDGEMENTS}

We would like to thank Dr. Safoora Zaminpardaz for developing the GNSS Research Centre RTK software. The combined broadcast ephemeris was obtained from the IGS MGEX campaign on CDDIS. Peter J.G. Teunissen is the recipient of an Australian Research Council (ARC) Federation Fellowship (Project Number FF0883188).

\section{DATA AVAILABILITY STATEMENT}

Some or all data, models, or code generated or used during the study are available in a repository or online in accordance with funder data retention policies (Combined broadcast ephemeris from the IGS MEGX campaign on CDDIS under MGEX (2018); see also Montenbruck et al. (2014) and Montenbruck et al. (2017)).

Some or all data, models, or code generated or used during the study are available from the corresponding author by request $(1 \mathrm{~Hz}$ RINEX observation files of the tested stations on the test days).

\section{REFERENCES}

Amiri-Simkooei, A. R., Teunissen, P. J. G., and Tiberius, C. C. J. M. (2009). "Application of least-squares variance component estimation to GPS observables." J. Surv. Eng., 135(4), 149-160, doi:10.1061/(ASCE)0733-9453(2009)135:4(149).

Betz, J. W. (2016). Engineering satellite-based navigation and timing: global navigation satellite systems, signals, and receivers. Wiley, IEEE Press, doi:10.1002/9781119141167.

Bogen, A. H. (1974). "Geometric performance of the global positioning system." Report No. SAMSO-Tit-74-169, Aerospace Corporation.

Euler, H. J. and Goad, C. C. (1991). "On optimal filtering of GPS dual frequency observations without using orbit information." Bull. Geod., 65(2), 130-143, doi:10.1007/BF00806368.

Geng, J. and Bock, Y. (2013). "Triple-frequency GPS precise point positioning with rapid ambiguity resolution." J. Geodesy, 87(5), 449-460, doi:10.1007/s00190-013-0619-2.

Gleason, S. and Gebre-Egziabher, D. (2009). GNSS: applications and methods. Artech House. ISRO (2018). "List of navigation satellites." Department of Space, Indian Space Research Organisation, <https://www.isro.gov.in/spacecraft/list-of-navigation-satellites $>$ (December, 2018).

Langley, R. B. (1999). "Dilution of precision." Innovation, GPS World, 10(5), 52-59.

Li, L., Li, Z., Yuan, H., Wang, L., and Hou, Y. (2016). "Integrity monitoring-based ratio test for GNSS integer ambiguity validation." GPS Solut., 20(3), 573-585, doi:10.1007/s10291015-0468-y.

Liu, X., Zhang, S., Zhang, Q., Ding, N., and Yang, W. (2019). "A fast satellite selection algorithm with floating high cut-off elevation angle based on ADOP for instantaneous multi-GNSS single-frequency relative positioning." Adv. Space Res., 63(3), 1234-1252, doi:10.1016/j.asr.2018.10.032.

Liu, X., Zhang, S., Zhang, Q., and Yang, W. (2017). "An extended ADOP for performance evaluation of single-frequency single-epoch positioning by BDS/GPS in Asia-Pacific region." Sensors, 17(10), 2254, doi:10.3390/s17102254. 
MGEX (2018). "MGEX combined broadcast ephemeris in 2018." CDDIS, <ftp://ftp.cddis.eosdis.nasa.gov/gnss/data/campaign/mgex/daily/rinex3/2018/brdm> (November, 2018).

Montenbruck, O., Steigenberger, P., Khachikyan, R., Weber, G., Langley, R. B., Mervart, L., and Hugentobler, U. (2014). "IGS-MGEX: preparing the ground for multi-constellation GNSS science." Inside GNSS, January/February 2014, 42-49.

Montenbruck, O., Steigenberger, P., Prange, L., Deng, Z., Zhao, Q., Perosanz, F., Romero, I., Noll, C., Stürze, A., Weber, G., Schmid, R., MacLeod, K., and Schaer, S. (2017). "The multi-GNSS experiment (MGEX) of the international GNSS service (IGS) - achievements, prospects and challenges." Adv. Space Res., 59(7), 1671-1697, doi:10.1016/j.asr.2017.01.011.

Odijk, D., Nadarajah, N., Zaminpardaz, S., and Teunissen, P. J. G. (2017). "GPS, Galileo, QZSS and IRNSS differential ISBs: estimation and application." GPS Solut., 21(2), 439450, doi:10.1007/s10291-016-0536-y.

Odijk, D. and Teunissen, P. J. G. (2008). "ADOP in closed form for a hierarchy of multifrequency single-baseline GNSS models." J. Geodesy, 82, 473-492, doi:10.1007/s00190007-0197-2.

Odolinski, R., Teunissen, P. J. G., and Odijk, D. (2015). "Combined BDS, Galileo, QZSS and GPS single-frequency RTK." GPS Solut., 19(1), 151-163, doi:10.1007/s10291-014-0376-6.

Spilker, J. J. (1996). "Satellite constellation and geometric dilution of precision." Global Positioning System: Theory and Applications, Volume I, American Institute of Aeronautics and Astronautics, Inc., Chapter 5, 177-208. doi:10.2514/5.9781600866388.0177.0208.

Teunissen, P. J. G. (1995). "The least-squares ambiguity decorrelation adjustment: a method for fast GPS integer ambiguity estimation." J. Geodesy, 70(1-2), 65-82, doi:10.1007/BF00863419.

Teunissen, P. J. G. (1997). "A canonical theory for short GPS baselines. Part IV: precision versus reliability." J. Geodesy, 71(9), 513-525, doi:10.1007/s001900050119.

Teunissen, P. J. G. (1998). "Success probability of integer GPS ambiguity rounding and bootstrapping." J. Geodesy, 72(10), 606-612, doi:10.1007/s001900050199.

Teunissen, P. J. G., de Jonge, P. J., and Tiberius, C. C. J. M. (1996). "The volume of the GPS ambiguity search space and its relevance for integer ambiguity resolution." Proc., ION GPS 1996, 889-898.

Teunissen, P. J. G. and Montenbruck, O. (2017). Springer handbook of global navigation satellite systems. Springer International Publishing, Cham, Switzerland, doi:10.1007/9783-319-42928-1.

Teunissen, P. J. G., Simons, D. G., and Tiberius, C. C. J. M. (2006). "Probability and observation theory." Lecture Notes AE2-E01, Faculty of Aerospace Engineering, Delft University of Technology.

Wang, K., Chen, P., Zaminpardaz, S., and Teunissen, P. J. G. (2019). "Precise regional L5 positioning with IRNSS and QZSS: stand alone and combined." GPS Solut., 23(1), 10, doi:10.1007/s10291-018-0800-4.

Zaminpardaz, S., Wang, K., and Teunissen, P. J. G. (2018). "Australia-first high-precision positioning results with new Japanese QZSS regional satellite system." GPS Solut., 22(4), 101, doi:10.1007/s10291-018-0763-5. 


\section{List of Tables}

1 Zenith-reference standard deviations for code $\left(\sigma_{p}\right)$ and phase $\left(\sigma_{\phi}\right)$ observations. For the computation and multipath mitigation, the day pairs DOY 325/326 and DOY 328/329, 2018 were used for analysis of the GPS signals for baselines CUAA-CUBB and CUCC-SPA7, respectively. Data on the day pairs DOY 345/346 and DOY 335/336, 2018 were used for analysis of the QZSS signals of baselines CUAA-CUBB and CUCC-SPA7, respectively. Data on the day pair 345/346 were used for analysis of the NAVIC signals for both baselines. . . . . . . . . . . . . . . . . . . .

2 Empirical and time-averaged formal standard deviations (in parentheses) for ambiguity-fixed baselines CUAA-CUBB and CUCC-SPA7. . . . . . . . . 14

3 Empirical and time-averaged formal ASRs for baselines CUAA-CUBB and CUCC-SPA7. The data on DOY 328/329 and DOY 325/326, 2018 were used for processing and multipath mitigation in GPS single- and multi-frequency scenarios for baselines CUAA-CUBB and CUCC-SPA7, respectively. The data on DOY 343/344, 2018 were used in the L5 multi-GNSS scenarios for both baselines. . . . . . . . . . . . . . . . . 
Table 1. Zenith-reference standard deviations for code $\left(\sigma_{p}\right)$ and phase $\left(\sigma_{\phi}\right)$ observations. For the computation and multipath mitigation, the day pairs DOY 325/326 and DOY 328/329, 2018 were used for analysis of the GPS signals for baselines CUAA-CUBB and CUCC-SPA7, respectively. Data on the day pairs DOY 345/346 and DOY 335/336, 2018 were used for analysis of the QZSS signals of baselines CUAA-CUBB and CUCC-SPA7, respectively. Data on the day pair 345/346 were used for analysis of the NAVIC signals for both baselines.

\begin{tabular}{c|c|c|c|c|c}
\hline System & Frequency & \multicolumn{2}{|c|}{ CUAA-CUBB } & \multicolumn{2}{c}{ CUCC-SPA7 } \\
\hline & & $\sigma_{p}(\mathrm{~cm})$ & $\sigma_{\phi}(\mathrm{mm})$ & $\sigma_{p}(\mathrm{~cm})$ & $\sigma_{\phi}(\mathrm{mm})$ \\
\hline GPS & L1 & 26 & 1 & 25 & 2 \\
\hline GPS & L2 & 23 & 1 & 24 & 2 \\
\hline GPS & L5 & 8 & 1 & 8 & 2 \\
\hline QZSS & L5 & 8 & 2 & 8 & 2 \\
\hline NAVIC & L5 & 29 & 1 & 29 & 2 \\
\hline
\end{tabular}


Table 2. Empirical and time-averaged formal standard deviations (in parentheses) for ambiguity-fixed baselines CUAA-CUBB and CUCC-SPA7.

\begin{tabular}{c|c|c|c|c|c|c|c}
\hline System & Frequency & \multicolumn{3}{|c|}{ CUAA-CUBB $(\mathrm{mm})$} & \multicolumn{3}{|c}{ CUCC-SPA7 $(\mathrm{mm})$} \\
\hline & & North & East & Height & North & East & Height \\
\hline GPS & L1 & $2(2)$ & $2(2)$ & $4(5)$ & $3(3)$ & $3(2)$ & $6(6)$ \\
\hline GPS & L1/L2 & $2(2)$ & $2(1)$ & $4(4)$ & $3(2)$ & $3(2)$ & $6(5)$ \\
\hline GPS & L1/L2/L5 & $2(1)$ & $2(1)$ & $4(3)$ & $3(2)$ & $3(2)$ & $6(4)$ \\
\hline GPS/QZSS & L5 & $5(4)$ & $11(11)$ & $13(12)$ & $6(5)$ & $12(12)$ & $14(14)$ \\
\hline GPS/NAVIC & L5 & $2(2)$ & $3(3)$ & $6(7)$ & $4(3)$ & $4(4)$ & $9(9)$ \\
\hline
\end{tabular}


Table 3. Empirical and time-averaged formal ASRs for baselines CUAA-CUBB and CUCCSPA7. The data on DOY 328/329 and DOY 325/326, 2018 were used for processing and multipath mitigation in GPS single- and multi-frequency scenarios for baselines CUAACUBB and CUCC-SPA7, respectively. The data on DOY 343/344, 2018 were used in the L5 multi-GNSS scenarios for both baselines.

\begin{tabular}{c|c|c|c|c|c}
\hline System & Frequency & \multicolumn{2}{|c|}{ CUAA-CUBB } & \multicolumn{2}{c}{ CUCC-SPA7 } \\
\hline & & Empirical & Formal & Empirical & Formal \\
\hline GPS & L1 & 0.925 & 0.924 & 0.872 & 0.878 \\
\hline GPS & L1/L2 & 1.000 & 1.000 & 1.000 & 1.000 \\
\hline GPS & L1/L2/L5 & 1.000 & 1.000 & 1.000 & 1.000 \\
\hline GPS/QZSS & L5 & 0.939 & 0.927 & 0.924 & 0.912 \\
\hline GPS/NAVIC & L5 & 0.984 & 0.983 & 0.973 & 0.974 \\
\hline
\end{tabular}




\section{List of Figures}

1 Skyplots of GPS satellites on DOY 328, 2018 (a) and GPS IIF, NAVIC and QZSS satellites on DOY 343, 2018 (b). Station CUAA was used for the skyplots. 18

2 The average ambiguity-fixed baseline precision (a) compared with its PDOPbased approximation (15) (b). The 15 data of baseline CUCC-SPA7 on DOY 343, 2018 were used for the plots. . . . . . . . . . . . . . . . . . . 19

3 PDOP and ADOP using GPS L1 signals. The data of baseline CUAA-CUBB on DOY 328, 2018 were used for the plots. Note that the circles and x-marks refer to ADOP as explained in the legend. . . . . . . . . . . . . . . .

$4 \quad$ PDOP and ADOP using GPS/QZSS (a) and GPS/NAVIC (b) combined L5 signals. The data of baseline CUCC-SPA7 on DOY 343, 2018 were used for the plots. Note that the scales of the subfigures are different for a better illustration. Time periods from $77949 \mathrm{~s}$ to $81050 \mathrm{~s}$ for (a) and from $20219 \mathrm{~s}$ to $26220 \mathrm{~s}$ for (b) were zoomed in (c) and (d), respectively. . . . . . . . .

5 North (a), east (b) and height (c) errors. At the left side of the y-axis, the dots (the first item in the legend), solid lines (the third item in the legend) and x-marks (the fourth item in the legend) represent the ambiguity-float, ambiguity-correctly-fixed and ambiguity-wrongly-fixed solutions, respectively. The dotted lines (the second item in the legend) illustrate the $95 \%$ formal confidence intervals. At the right side of the y-axis, the ADOPs (the fifth item in the legend) are illustrated as pentagrams with 0.12 cycles marked with dashed lines. L5 signals of GPS/NAVIC satellites for baseline CUCCSPA7 on DOY 343, 2018 were used for the plots. The data on DOY 344, 2018 were used for multipath mitigation. . . . . . . . . . . . . 
6 North (a), east (b) and height (c) errors. At the left side of the y-axis, the dots (the first item in the legend), solid lines (the third item in the legend) and $\mathrm{x}$-marks (the fourth item in the legend) represent the ambiguity-float, ambiguity-correctly-fixed and ambiguity-wrongly-fixed solutions, respectively. The dotted lines illustrate the $95 \%$ formal confidence intervals. At the right side of the y-axis, the ADOPs are illustrated as pentagrams (the fifth item in the legend) with 0.12 cycles marked with dashed lines. L1 signals of GPS satellites for baseline CUAA-CUBB on DOY 328, 2018 were used for the plots. The data on DOY 329, 2018 were used for multipath mitigation. . . . . . . .

7 North (a), east (b) and height (c) errors in the ambiguity-fixed case. At the left side of the y-axis, the dots (the first item in the legend) and the dotted lines (the second item in the legend) illustrate the ambiguity-correctly-fixed solutions and their $95 \%$ formal confidence intervals. At the right side of the yaxis, the PDOPs are illustrated as pentagrams (the third item in the legend). L5 signals of GPS/NAVIC satellites for baseline CUCC-SPA7 on DOY 343, 2018 were used for the plots. Data on DOY 344, 2018 were used for multipath

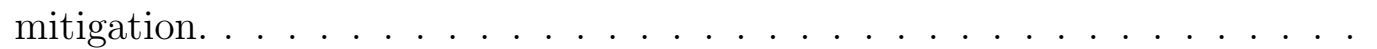

8 North (a), east (b) and height (c) errors in the ambiguity-fixed case. At the left side of the y-axis, the dots (the first item in the legend) and the dotted lines (the second item in the legend) illustrate the ambiguity-correctly-fixed solutions and their $95 \%$ formal confidence intervals. At the right side of the $y$ axis, the PDOPs are illustrated as pentagrams (the third item in the legend). GPS L1 signals for baseline CUAA-CUBB on DOY 328, 2018 were used for the plots. Data on DOY 329, 2018 were used for multipath mitigation. . . 


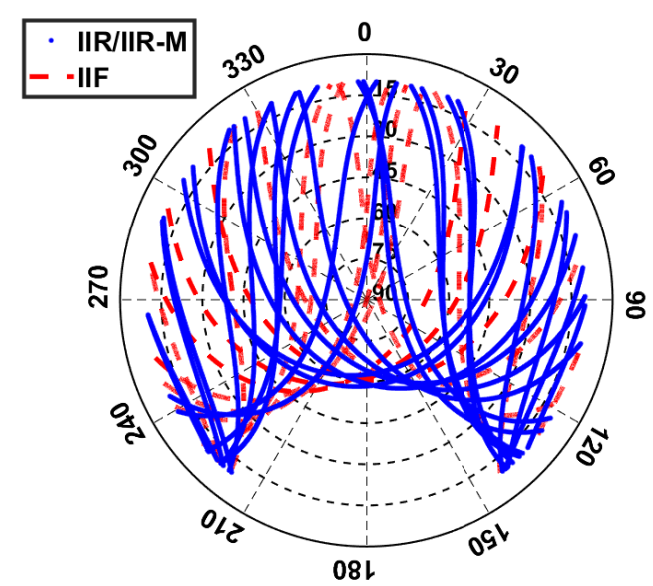

(a) GPS

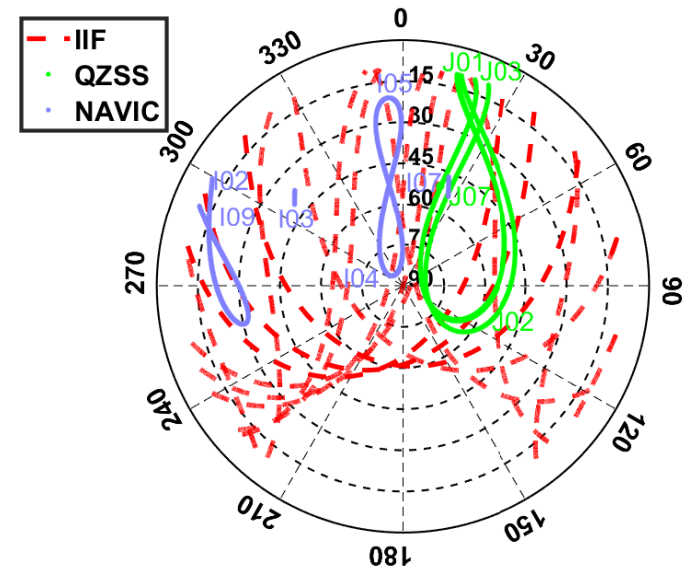

(b) GPS IIF, NAVIC, QZSS

Fig. 1. Skyplots of GPS satellites on DOY 328, 2018 (a) and GPS IIF, NAVIC and QZSS satellites on DOY 343, 2018 (b). Station CUAA was used for the skyplots. 


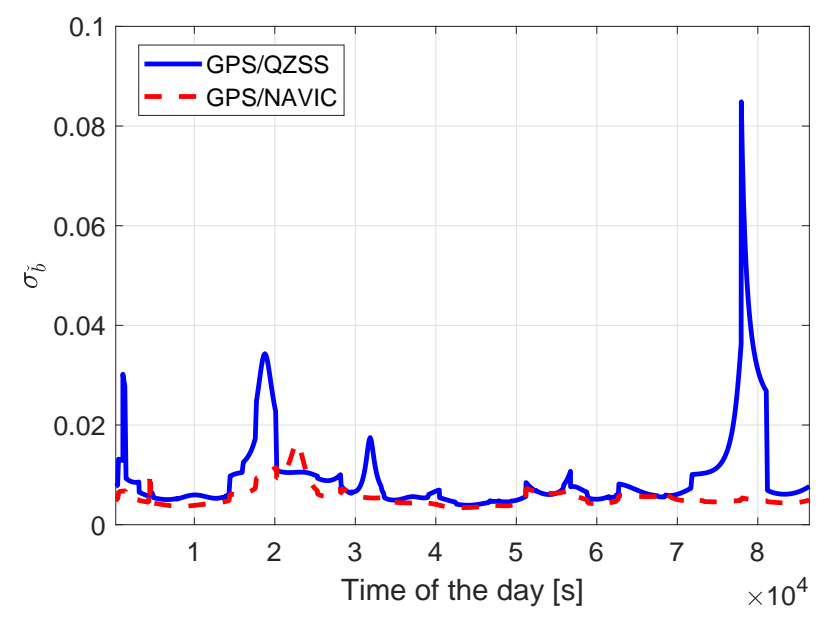

(a) $\sigma_{\breve{b}}$

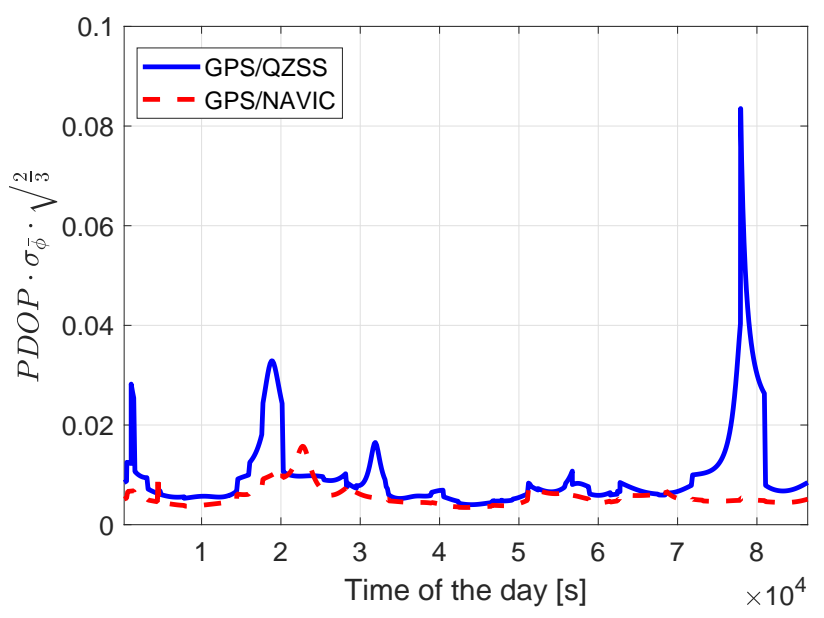

(b) $P D O P \cdot \sigma_{\bar{\phi}} \cdot \sqrt{\frac{2}{3}}$

Fig. 2. The average ambiguity-fixed baseline precision (a) compared with its PDOP-based approximation (15) (b). The 15 data of baseline CUCC-SPA7 on DOY 343, 2018 were used for the plots. 


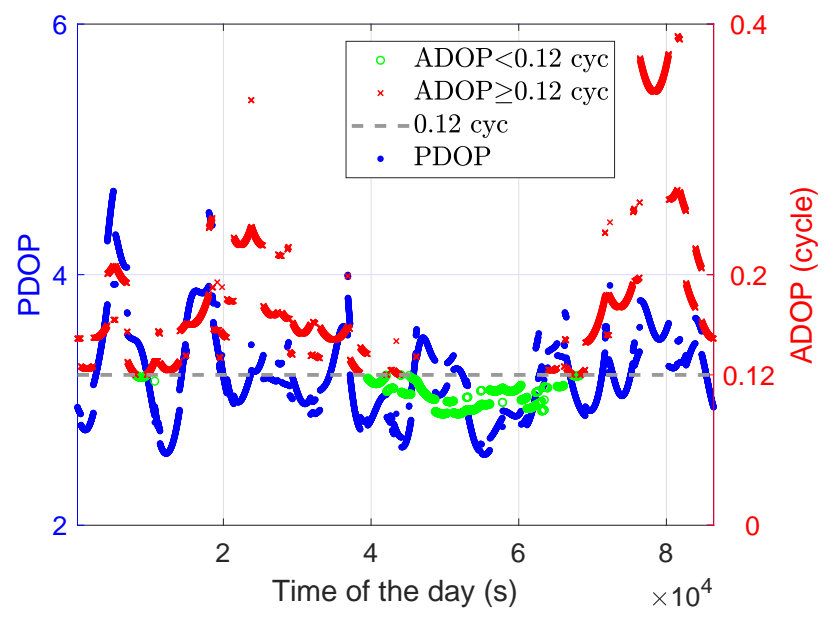

Fig. 3. PDOP and ADOP using GPS L1 signals. The data of baseline CUAA-CUBB on DOY 328, 2018 were used for the plots. Note that the circles and x-marks refer to ADOP as explained in the legend. 


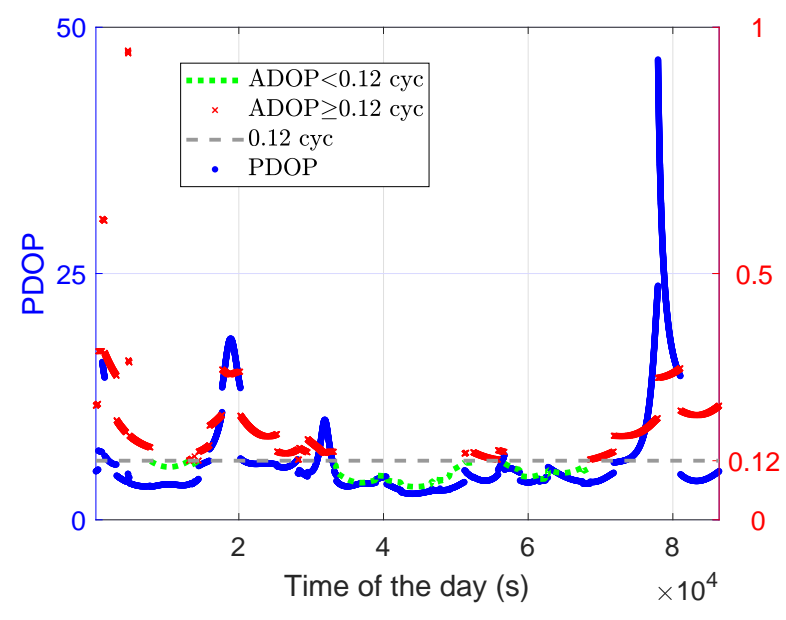

(a) GPS/QZSS

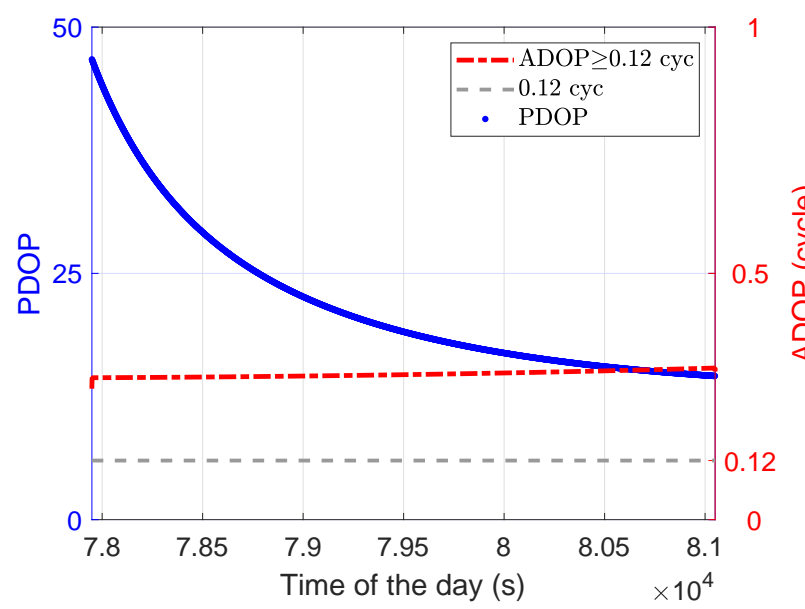

(c) GPS/QZSS (zoomed in)

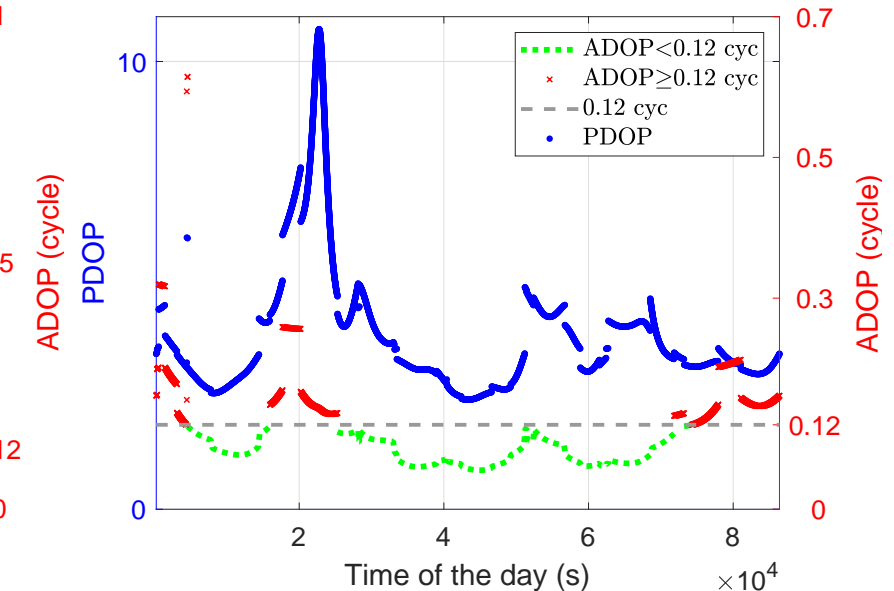

(b) GPS/NAVIC

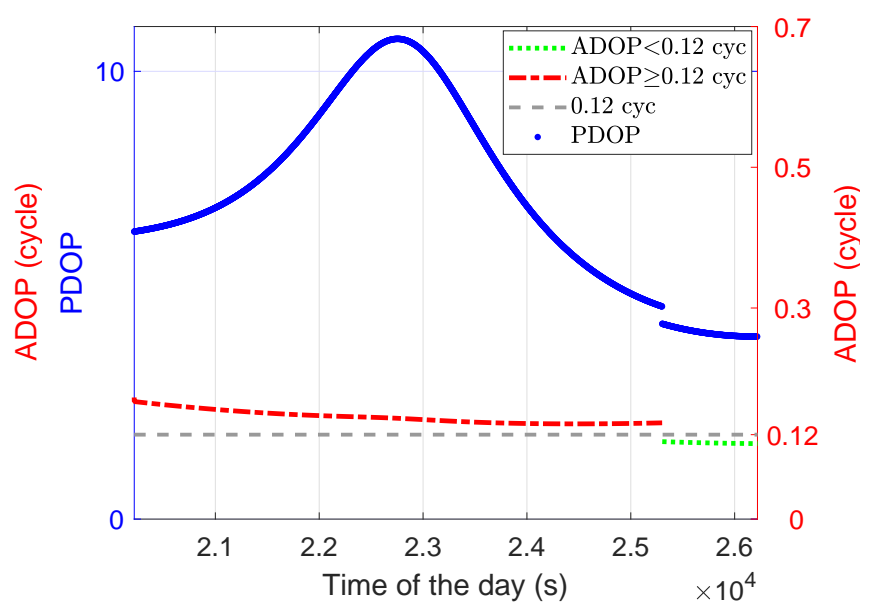

(d) GPS/NAVIC (zoomed in)

Fig. 4. PDOP and ADOP using GPS/QZSS (a) and GPS/NAVIC (b) combined L5 signals. The data of baseline CUCC-SPA7 on DOY 343, 2018 were used for the plots. Note that the scales of the subfigures are different for a better illustration. Time periods from $77949 \mathrm{~s}$ to $81050 \mathrm{~s}$ for (a) and from $20219 \mathrm{~s}$ to $26220 \mathrm{~s}$ for (b) were zoomed in (c) and (d), respectively. 


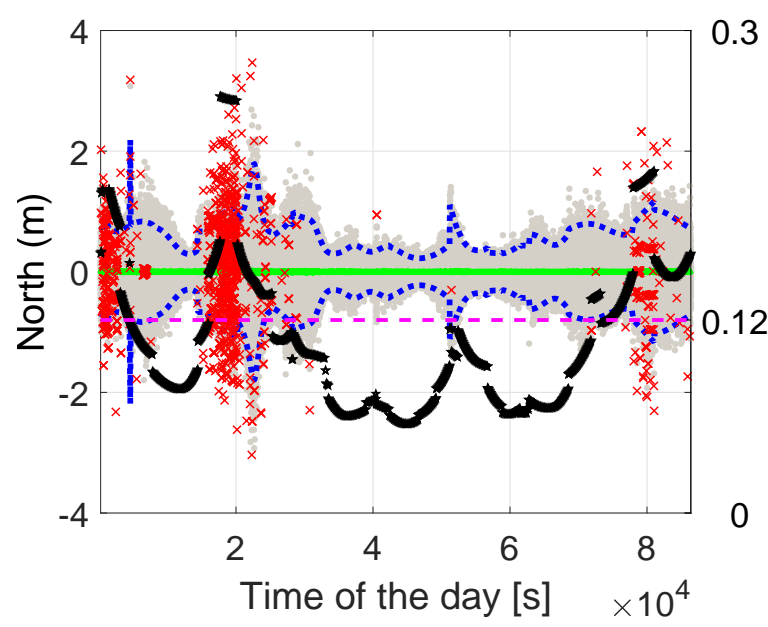

(a) North

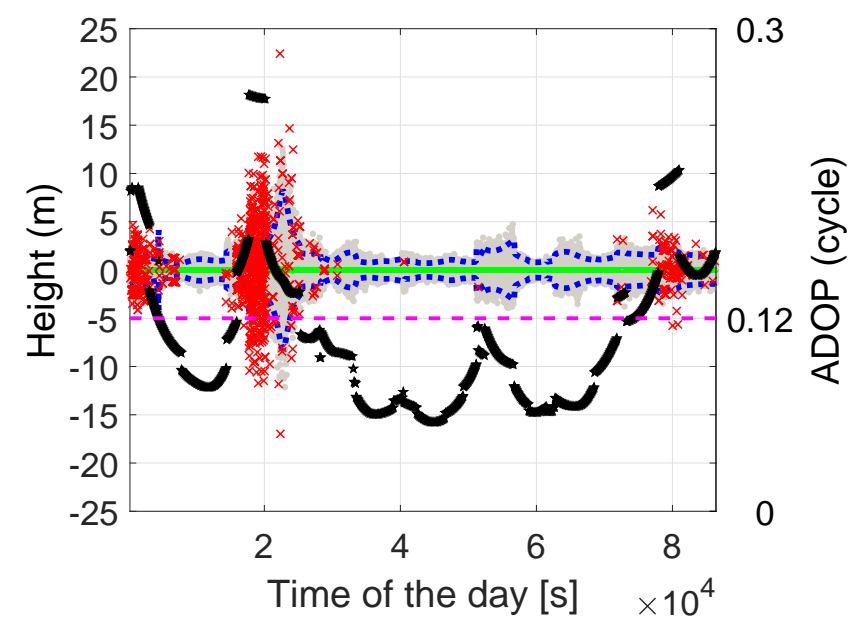

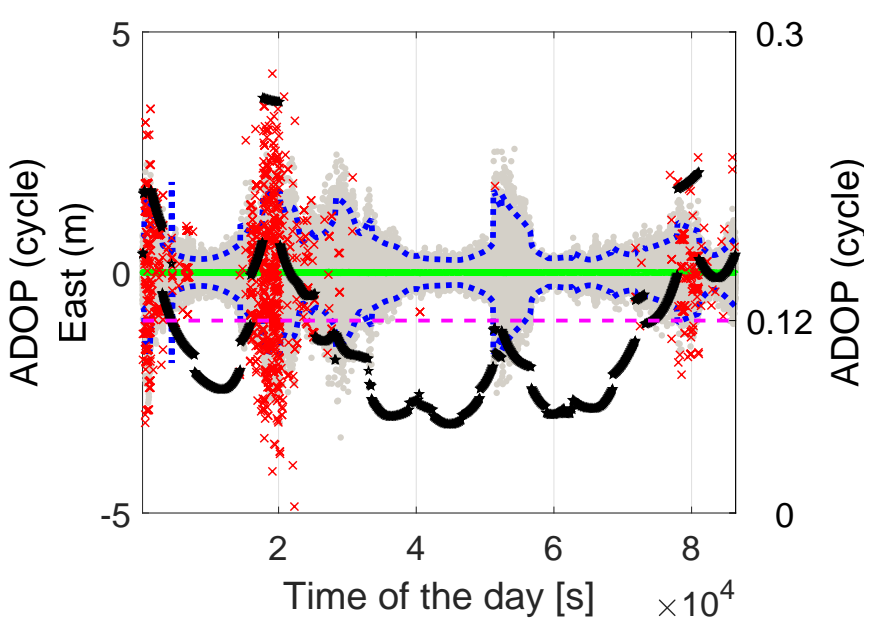

(b) East

\section{(c) Height}

Fig. 5. North (a), east (b) and height (c) errors. At the left side of the y-axis, the dots (the first item in the legend), solid lines (the third item in the legend) and x-marks (the fourth item in the legend) represent the ambiguity-float, ambiguity-correctly-fixed and ambiguity-wrongly-fixed solutions, respectively. The dotted lines (the second item in the legend) illustrate the $95 \%$ formal confidence intervals. At the right side of the y-axis, the ADOPs (the fifth item in the legend) are illustrated as pentagrams with 0.12 cycles marked with dashed lines. L5 signals of GPS/NAVIC satellites for baseline CUCC-SPA7 on DOY 343, 2018 were used for the plots. The data on DOY 344, 2018 were used for multipath mitigation. 


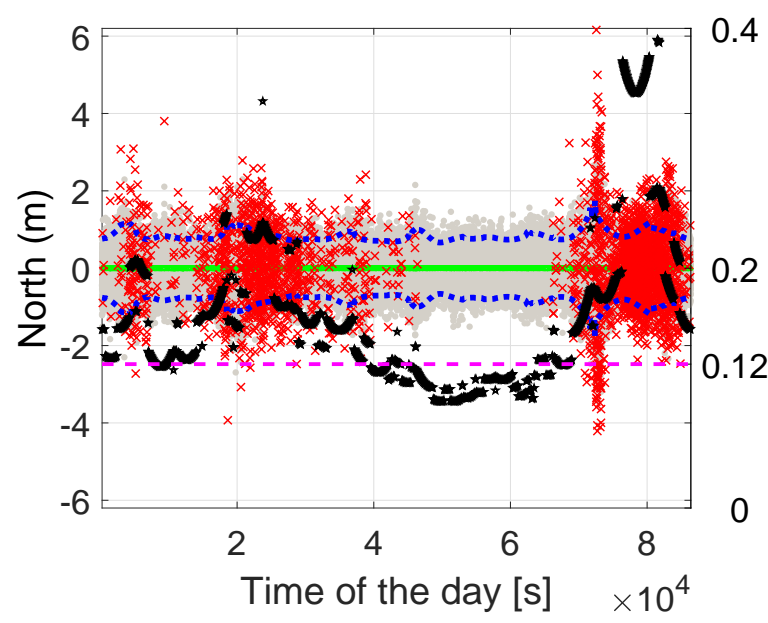

(a) North

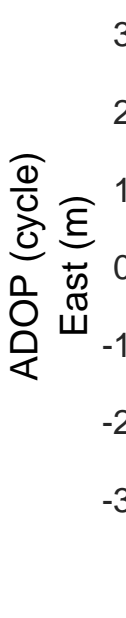

0.4

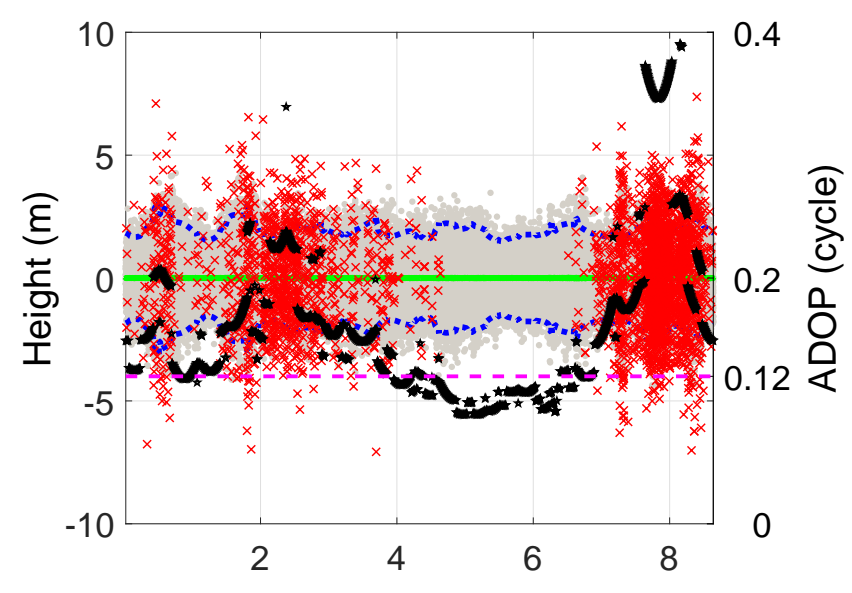

(c) Height

Fig. 6. North (a), east (b) and height (c) errors. At the left side of the y-axis, the dots (the first item in the legend), solid lines (the third item in the legend) and x-marks (the fourth item in the legend) represent the ambiguity-float, ambiguity-correctly-fixed and ambiguitywrongly-fixed solutions, respectively. The dotted lines illustrate the $95 \%$ formal confidence intervals. At the right side of the y-axis, the ADOPs are illustrated as pentagrams (the fifth item in the legend) with 0.12 cycles marked with dashed lines. L1 signals of GPS satellites for baseline CUAA-CUBB on DOY 328, 2018 were used for the plots. The data on DOY 329, 2018 were used for multipath mitigation. 


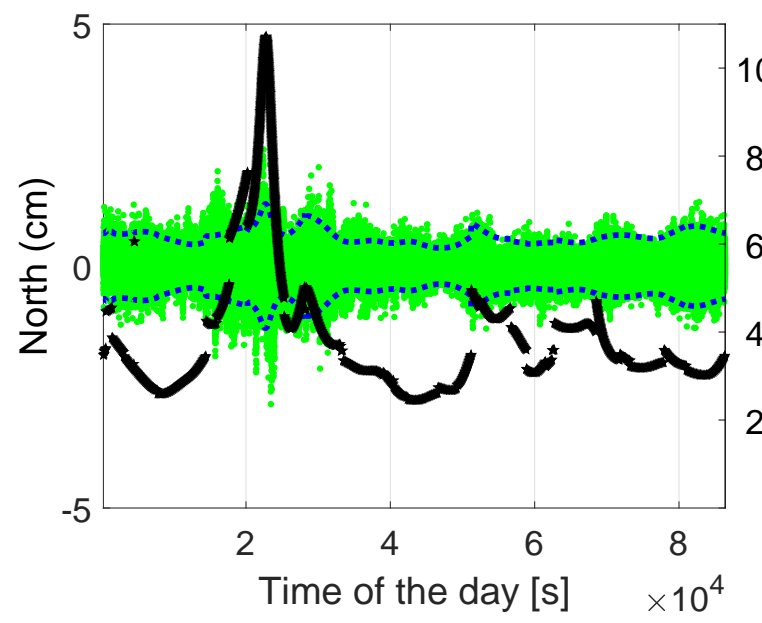

(a) North

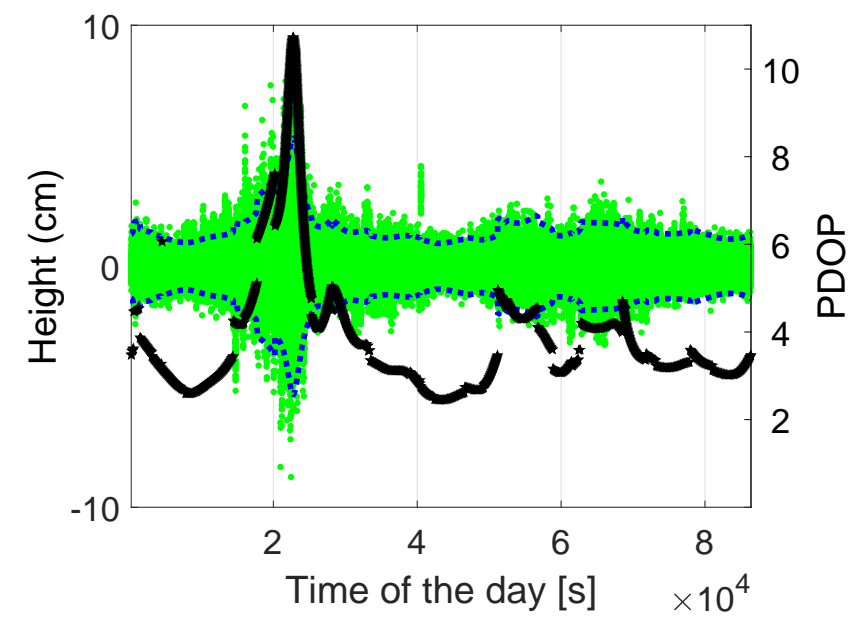

(c) Height

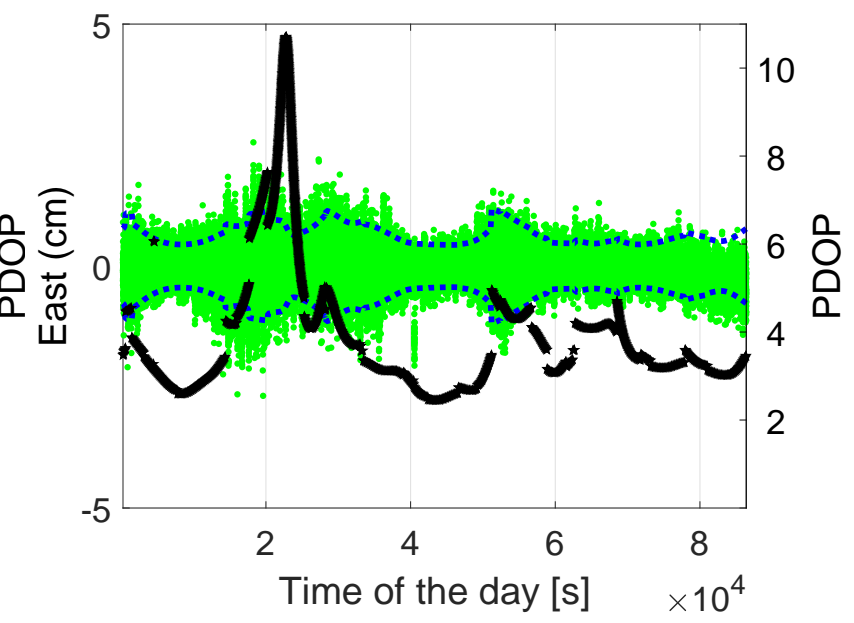

(b) East

Fig. 7. North (a), east (b) and height (c) errors in the ambiguity-fixed case. At the left side of the y-axis, the dots (the first item in the legend) and the dotted lines (the second item in the legend) illustrate the ambiguity-correctly-fixed solutions and their $95 \%$ formal confidence intervals. At the right side of the y-axis, the PDOPs are illustrated as pentagrams (the third item in the legend). L5 signals of GPS/NAVIC satellites for baseline CUCC-SPA7 on DOY 343, 2018 were used for the plots. Data on DOY 344, 2018 were used for multipath mitigation. 


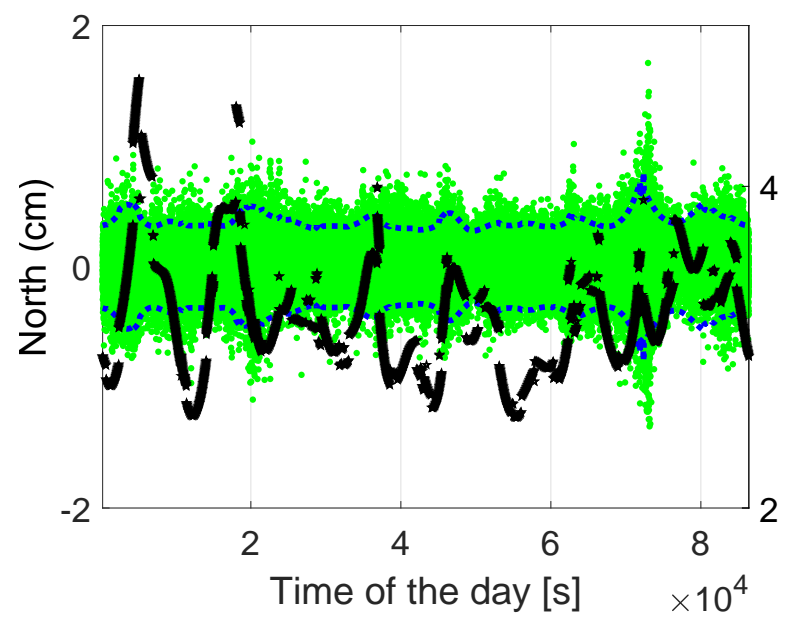

(a) North

(c) Height

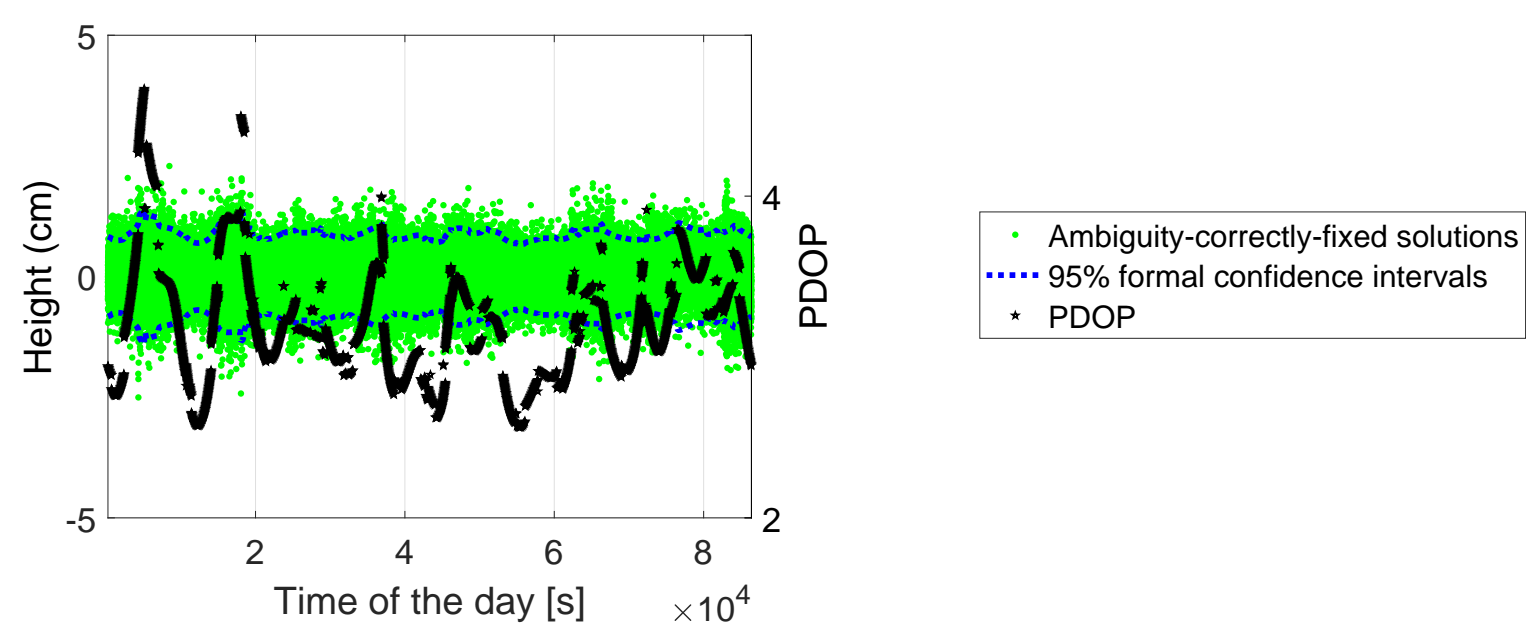

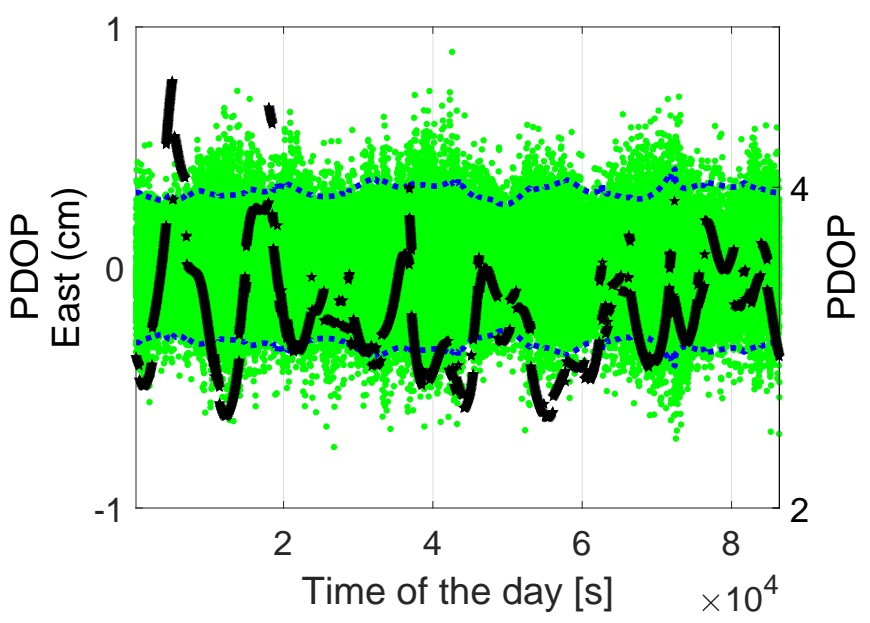

(b) East

Fig. 8. North (a), east (b) and height (c) errors in the ambiguity-fixed case. At the left side of the y-axis, the dots (the first item in the legend) and the dotted lines (the second item in the legend) illustrate the ambiguity-correctly-fixed solutions and their $95 \%$ formal confidence intervals. At the right side of the y-axis, the PDOPs are illustrated as pentagrams (the third item in the legend). GPS L1 signals for baseline CUAA-CUBB on DOY 328, 2018 were used for the plots. Data on DOY 329, 2018 were used for multipath mitigation. 